MATHEMATICS OF COMPUTATION

Volume 73, Number 247, Pages 1385-1392

S 0025-5718(04)01639-4

Article electronically published on February 18, 2004

\title{
A STABLE TEST TO CHECK IF A MATRIX IS A NONSINGULAR $M$-MATRIX
}

\author{
J. M. PEÑA
}

\begin{abstract}
A stable test for checking if a matrix is a nonsingular $M$-matrix is presented. Its computational cost is, in the worst case, $O\left(n^{2}\right)$ elementary operations higher than the computational cost of Gaussian elimination. The test can be applied to check if a nonnegative matrix has spectral radius less than 1 .
\end{abstract}

\section{INTRODUCTION AND BASIC NOTATIONS}

A real matrix with nonpositive off-diagonal elements is called a $Z$-matrix. Nonsingular $M$-matrices form a subclass of $Z$-matrices and have important applications; for instance, in iterative methods in numerical analysis, in the analysis of dynamical systems, in economics and in mathematical programming. Nonsingular $M$-matrices have many equivalent definitions. In fact, in Theorem 2.3 in Chapter 6 of [2] there appear 50 equivalent definitions. In particular, given a $Z$-matrix, the following three properties are equivalent: $A$ is a nonsingular $M$-matrix, $A^{-1}$ is nonnegative and all leading principal minors of $A$ are strictly positive. Clearly, a triangular $Z$-matrix is a nonsingular $M$-matrix if and only if it has positive diagonal entries. Since the positivity of the leading principal minors is a necessary and sufficient condition to ensure that a $Z$-matrix is a nonsingular $M$-matrix, a test to check if a nontriangular matrix is a nonsingular $M$-matrix consists of performing Gaussian elimination and checking if the pivots are positive. It is well known that the growth factor is an indicator of stability (cf. 4, 5]). Unfortunately, the growth factor of the mentioned possible test can be arbitrarily large even for $3 \times 3 M$-matrices, as Example 3.2 shows. On the other hand, it is also known that strict diagonal dominance by rows with positive diagonal entries or irreducibility joint with diagonal dominance by rows with positive diagonal entries are sufficient conditions to assure that a $Z$-matrix is a nonsingular $M$-matrix (see (6.2.17) of [7]) and each of both properties requires $O\left(n^{2}\right)$ elementary operations to be applied. But if we have to check these conditions at each step of Gaussian elimination, it would add $O\left(n^{3}\right)$ elementary operations to the computational cost of Gaussian elimination.

In Section 2 we present a test to check if an $n \times n Z$-matrix is a nonsingular $M$-matrix such that the corresponding growth factor is bounded above by $n-1$ (as proved in Theorem 3.1) and, simultaneously, it applies (if possible) the mentioned

Received by the editor January 11, 2002 and, in revised form, January 4, 2003.

2000 Mathematics Subject Classification. Primary 65F30, 65F05, 65G99.

Key words and phrases. Test for $M$-matrices, diagonal dominance, stability, growth factor.

This research has been partially supported by the Spanish Research Grant CICYT BFM20001253.

(C)2004 American Mathematical Society 
sufficient conditions at each step of Gaussian elimination. In the worst case, our test adds $O\left(n^{2}\right)$ elementary operations to the computational cost of Gaussian elimination and it leads to an upper triangular matrix strictly diagonally dominant by rows. In [8] it was shown that a pivoting strategy leading to an upper triangular matrix diagonally dominant by rows has more advantages than when it leads to an upper triangular matrix diagonally dominant by columns. This has been confirmed again for Gauss-Jordan elimination in 6]. In Section 2 we also include an application of our test to check if a nonnegative matrix has spectral radius less than 1.

The growth factor of a numerical algorithm is usually defined as the quotient between the maximal absolute value of all the elements that occur during the performance of the algorithm and the maximal absolute value of all the initial data. A remarkable property of our test is that it has a controlled growth factor even when it is applied to a $Z$-matrix which is not an $M$-matrix. Let us compare this behavior with the well-known test to check if a symmetric matrix is definite positive, which uses the signs of the pivots of Gaussian elimination. If the matrix is definite positive, then it is known that the growth factor is 1 . But, if we consider a symmetric matrix which is not definite positive, then the growth factor of the computed elements can be arbitrarily large, as the following matrix shows:

$$
\left(\begin{array}{ccc}
\varepsilon & \varepsilon & 1 \\
\varepsilon & 2 \varepsilon & 0 \\
1 & 0 & 1
\end{array}\right)
$$

$\left(0<\varepsilon<\frac{1}{2}\right)$. After one step of Gauss elimination we obtain the matrix

$$
\left(\begin{array}{ccc}
\varepsilon & \varepsilon & 1 \\
0 & \varepsilon & -1 \\
0 & -1 & 1-\frac{1}{\varepsilon}
\end{array}\right),
$$

and the growth factor corresponding to this step is $\left|1-\frac{1}{\varepsilon}\right|$.

\section{Characterization and test}

Given $k \in\{1,2, \ldots, n\}$, let $\alpha$ be any increasing sequence of $k$ positive integers less than or equal to $n$. Then we denote by $A[\alpha]$ the $k \times k$ submatrix of $A$ containing rows and columns numbered by $\alpha$. If $c \in \mathbf{R}^{n}, c \geq 0$ (resp., $\leq 0$ ) means that all components of $c$ are nonnegative (resp., nonpositive) and $c>0$ (resp., $<0$ ) means that all components of $c$ are positive (resp., negative). Gaussian elimination (GE) transforms a linear system $M x=b$ into an equivalent upper triangular linear system $U x=c$. Gaussian elimination with a given pivoting strategy, for nonsingular matrices $M$, consists of a succession of at most $n-1$ major steps resulting in a sequence of matrices:

$$
M=M^{(1)} \longrightarrow \tilde{M}^{(1)} \longrightarrow M^{(2)} \longrightarrow \tilde{M}^{(2)} \longrightarrow \cdots \longrightarrow M^{(n)}=\tilde{M}^{(n)}=U,
$$

where $M^{(t)}=\left(m_{i j}^{(t)}\right)_{1 \leq i, j \leq n}$ has zeros below its main diagonal in the first $t-1$ columns. The same transformations should be carried out in the vector $b$ :

$$
b=b^{(1)} \rightarrow \tilde{b}^{(1)} \rightarrow b^{(2)} \rightarrow \tilde{b}^{(2)} \rightarrow \cdots \rightarrow b^{(n)}=\tilde{b}^{(n)}=c .
$$

The matrix $\tilde{M}^{(t)}=\left(\tilde{m}_{i j}^{(t)}\right)_{1 \leq i, j \leq n}$ is obtained from the matrix $M^{(t)}$ by reordering the rows and/or columns $t, t+1, \ldots, n$ of $M^{(t)}$ according to the given pivoting 
strategy and satisfying $\tilde{m}_{t t}^{(t)} \neq 0$. To obtain $M^{(t+1)}$ from $\tilde{M}^{(t)}$, we produce zeros in column $t$ below the pivot element $\tilde{m}_{t t}^{(t)}$ by subtracting multiples of row $t$ from the rows beneath it. If $P$ and/or $Q$ are permutation matrices such that the Gaussian elimination of $B=P M Q$ can be performed without row and column exchanges, then the entry $(1,1)$ of $\tilde{M}^{(t)}[t, \ldots, n]$ coincides with the same entry of $B^{(t)}[t, \ldots, n]$. If $B=P^{T} M P$ (and so we change at the $t$ th step the $t$ th row and column by row and column $i_{t}$ ), we say that we have performed symmetric pivoting.

Let us define $e_{n} \in \mathbf{R}^{n}$ by

$$
e_{n}=(1, \ldots, 1)^{T} .
$$

Theorem 2.1 (i) provides the characterization of nonsingular $M$-matrices which will be used in the test.

Theorem 2.1. Let $M=\left(m_{i j}\right)_{1 \leq i, j \leq n}$ be a $Z$-matrix and $b:=M e_{n}$, where $e_{n}$ is defined in (2.3). For each $k=1, \ldots, n$, let $M^{(k)}=\left(m_{i j}^{(k)}\right)_{1 \leq i, j \leq n}, b^{(k)}=\left(b_{i}^{(k)}\right)_{1 \leq i \leq n}$ be the matrices and vectors (see (2.1) and (2.2), respectively) obtained after $k$ steps of $G E$ of $M x=b$ with symmetric pivoting, choosing at each step as pivot index the first index $i_{k} \in\{k, \ldots, n\}$ such that $b_{i_{k}}^{(k)}=\max _{k \leq j \leq n}\left\{b_{j}^{(k)}\right\}$. Then:

(i) $M$ is a nonsingular $M$-matrix if and only if

$$
b_{i_{k}}^{(k)}>0, \quad k=1, \ldots, n .
$$

(ii) Let $k \in\{1, \ldots, n-1\}$ be such that $b_{i_{r}}^{(r)}>0$ for all $r<k$. If $M^{(k)}$ is upper triangular with positive diagonal elements, then $M$ is a nonsingular $M$-matrix and if either $M^{(k)}$ is upper triangular with nonnegative diagonal elements or $b^{(k)}[k, \ldots, n] \geq 0$, then $M$ is an $M$-matrix. If, in the last case, either $b^{(k)}[k, \ldots, n]>0$ or we have the condition $b_{i_{k}}^{(k)}>0$ and $m_{i j}^{(k)} \neq 0$ for all $i, j \geq k$ with $|i-j|=1$, then $M$ is a nonsingular $M$-matrix.

Proof. (i) Let us assume that $M$ is a nonsingular $M$-matrix and let us prove that (2.4) holds. Then, applying Theorem 2 of [1] to $M^{T}, M$ has a row which is strictly diagonally dominant. Therefore, $b^{(1)}=b=M e_{n}$ has a positive component and so $b_{i_{1}}^{(1)}>0$. Let $P$ be the permutation matrix obtained from the identity matrix by interchanging rows 1 and $i_{1}$. Then $\tilde{M}^{(1)}=P M P^{T}$ is again a nonsingular $M$-matrix and $P b=\left(P M P^{T}\right) P e_{n}=\left(P M P^{T}\right) e_{n}$. If we perform a step of GE to $\left(P M P^{T}\right) x=$ $P b$, we obtain a matrix $M^{(2)}$ and $M^{(2)}[2, \ldots, n]$ is again a nonsingular $M$-matrix because such property is inherited by Schur complements (cf. [3]). Therefore, we again know that $M^{(2)}[2, \ldots, n]$ has a row which is strictly diagonally dominant. Since $M^{(2)} e_{n}=b^{(2)}$, we also have $M^{(2)}[2, \ldots, n] e_{n-1}=b^{(2)}[2, \ldots, n]$ and so the vector $b^{(2)}[2, \ldots, n]$ has a positive component. Then $b_{i_{2}}^{(2)}>0$ and, iterating the previous arguments, we derive (2.4).

Let us now assume that (2.4) holds and let us prove that $M$ is a nonsingular $M$-matrix. Let $P$ be the permutation matrix obtained from the identity matrix by reordering its rows according to (2.4). Then $\left(P M P^{T}\right) x=c$ (with $c:=P b$ ) has solution $P e_{n}=e_{n}$, its coefficient matrix $B:=P M P^{T}$ is a $Z$-matrix and, if we perform GE of $B$ without row or column exchanges, we obtain the equivalent systems

$$
B^{(k)} x=c^{(k)}
$$


and

$$
c^{(k)}=P b^{(k)}
$$

By $(2.4), c_{k}^{(k)}=b_{i_{k}}^{(k)}>0$ for all $k$. Since all systems (2.5) have solution $e_{n}$, the sum of the elements of each row of $B^{(k)}$ is given by the corresponding component of $c^{(k)}$. Since $B=B^{(1)}$ is a $Z$-matrix and the sum of the elements of its first row is $c_{1}^{(1)}=b_{i_{1}}^{(1)}>0$, we conclude that the $(1,1)$ entry of $B$ (and so its first pivot) is positive. Taking into account the sign of the elements of the first column of $B$, when we perform the first step of GE we are adding to each row a nonnegative multiple of the first row. Therefore $B^{(2)}$ is again a $Z$-matrix. Since the sum of the elements of its second row is $c_{2}^{(2)}=b_{i_{2}}^{(2)}>0$, we deduce that the second pivot of the $\mathrm{GE}$ of $B$ is also positive. Iterating the previous reasoning, we conclude that all matrices $B^{(k)}$ are $Z$-matrices and all pivots of the GE of $B$ are positive. Thus, $B=P M P^{T}$ is a $Z$-matrix whose leading principal minors are positive and so, by Theorem 2.3 of Chapter 6 of [2], it is a nonsingular $M$-matrix. Hence $M$ is also a nonsingular $M$-matrix.

(ii) Let $B:=P^{T} M P$, where $P$ is the permutation matrix obtained from the identity by interchanging rows $1, \ldots, k-1$ by rows $i_{1}, \ldots, i_{k-1}$, respectively, and let $c:=P b$. By using the arguments given above in the proof of the converse of the characterization, we can deduce from $b_{i_{r}}^{(r)}>0$ for all $r<k$ that the first $k-1$ pivots of the GE of $B$ are positive and that $B^{(k)}$ is a $Z$-matrix. In addition $B^{(k)} e_{n}=c^{(k)}$, and so

$$
B^{(k)}[k, \ldots, n] e_{n-k+1}=c^{(k)}[k, \ldots, n] .
$$

If $b^{(k)}[k, \ldots, n] \geq 0$, we deduce from (2.6) and the hypothesis that $c^{(k)}[k, \ldots, n] \geq 0$. If $B^{(k)}$ is upper triangular with positive diagonal elements, then all pivots of the GE of $B$ are positive and we again can deduce that $M$ is a nonsingular $M$-matrix. If all components of $b^{(k)}[k, \ldots, n]$ are positive then, again by (2.6) and the hypothesis, the same property holds for $c^{(k)}[k, \ldots, n]$. From $(2.7)$ we conclude that the sum of the elements of every row of $B^{(k)}[k, \ldots, n]$ is positive; that is, $B^{(k)}[k, \ldots, n]$ is a $Z$-matrix strictly diagonally dominant by rows with positive diagonal elements and so it is well known that all pivots of its GE are positive. In this case, all pivots of the GE of $B$ are positive and, reasoning as above, we deduce that $M$ is a nonsingular $M$-matrix. Let us now prove that $M$ (or, equivalently, $B$ ) is an $M$-matrix provided that either $M^{(k)}$ is upper triangular with nonnegative diagonal elements or $b^{(k)}[k, \ldots, n] \geq 0$. In fact, if $I_{r}$ is the $r \times r$ identity matrix, by Lemma 4.1 of Chapter 6 of [2], it is sufficient to see that $F_{\varepsilon}:=B^{(k)}[k, \ldots, n]+\varepsilon I_{n-k+1}$ is a nonsingular $M$-matrix for all $\varepsilon>0$. This is a consequence of the fact that $F_{\varepsilon}$ is upper triangular with positive diagonal elements in the first case, and of (6.2.17) of [7] and the fact that $F_{\varepsilon}$ is a $Z$-matrix strictly diagonally dominant by rows with positive diagonal elements for all $\varepsilon>0$ in the second case.

Finally, if $b^{(k)}[k, \ldots, n] \geq 0$ and $b_{i_{k}}^{(k)}>0$, then $c^{(k)}[k, \ldots, n] \geq 0$ and has at least a positive component, which implies by $(2.7)$ that $B^{(k)}[k, \ldots, n] e_{n-k+1} \geq 0$ with at least a positive component. If the elements $m_{i j}^{(k)} \neq 0$ for all $i, j \geq k$ with $|i-j|=1$, then the same property holds for the corresponding elements of $B^{(k)}[k, \ldots, n]$ and we can derive the irreducibility of this last matrix. Then, by (6.2.17) of [7, we conclude that $B^{(k)}[k, \ldots, n]$ is a nonsingular $M$-matrix and, so, the pivots of its 
GE are positive and we get the positivity of all pivots of the GE of $B$, concluding again that $M$ is a nonsingular $M$-matrix.

The following remark uses the previous result in order to construct a stable algorithm for checking if a given $Z$-matrix is a nonsingular $M$-matrix.

Algorithm 2.2. In order to know if a given matrix $M$ is a nonsingular $M$-matrix, we can derive an algorithm from Theorem 2.1 consisting of the following steps:

(I) If $M^{(1)}:=M$ is a triangular matrix, then $M$ is a nonsingular $M$-matrix if and only if it is a Z-matrix with all its diagonal entries positive. If $M^{(1)}$ is not a triangular matrix, then let $b^{(1)}:=M^{(1)} e_{n}$ (with $e_{n}$ defined in (2.3)).

(II) Apply the pivoting strategy indicated in Theorem 2.1 and calculate the vectors $b^{(k)}$ and matrices $M^{(k)}$ of (2.2) and (2.1) (starting with $b^{(1)}, b^{(2)}, M^{(2)}$ ) until when we find the first index $k \in\{1, \ldots, n\}$ such that one of the following cases appear:

(II.A) If $b^{(k)}[k, \ldots, n] \leq 0$, then $M$ is not a nonsingular $M$-matrix.

(II.B) If $b^{(k)}[k, \ldots, n]>0$, then $M$ is a nonsingular $M$-matrix.

(II.C) If $M^{(k)}$ is an upper triangular matrix with positive diagonal entries, then $M$ is a nonsingular $M$-matrix.

(II.D) If $b^{(k)}[k, \ldots, n] \geq 0$, it has at least a positive component and $M^{(k)}$ satisfies that $m_{i j}^{(k)} \neq 0$ for all $i, j \geq k$ with $|i-j|=1$, then $M$ is a nonsingular $M$-matrix.

When applying the previous algorithm, if we find $k$ such that $b_{i_{r}}^{(r)}>0$ for all $r<k$ and either $b^{(k)}[k, \ldots, n] \geq 0$ or $M^{(k)}$ is upper triangular with nonnegative diagonal entries, then we already know by Theorem 2.2 that $M$ is an $M$-matrix.

Let us consider the computational cost of the test provided in Algorithm 2.2. If it stops after calculating $b^{(k)}$, then it adds $k(k-1) / 2$ comparisons, $\left(2 n^{2}+(2 n-k-1) k\right) / 2$ sums, $(2 n-k-1) k / 2$ multiplications and $n-k+1$ divisions to the computational cost of obtaining the first $k-1$ pivots of GE without row exchanges. If it stops after calculating $M^{(k)}$, then it adds $k(k-1) / 2$ comparisons, $\left(2 n^{2}+(2 n-k-1) k\right) / 2$ sums and $(2 n-k-1) k / 2$ multiplications to the computational cost of obtaining the first $k$ pivots of GE without row exchanges. In the worst case $(k=n)$, it adds $O\left(n^{2}\right)$ elementary operations to the $O\left(n^{3}\right)$ elementary operations necessary for calculating the pivots of GE.

We finish this section by commenting on an important application of the previous test. In many fields it is very important to know if a given matrix $A$ has the spectral radius $\rho(A)<1$. It is well known (cf. Lemma 2.1 of Chapter 6 of [2]) that a nonnegative matrix $B$ satisfies $\rho(B)<1$ if and only if $I-B$ is a nonsingular $M$-matrix. Therefore, in order to check if $\rho(B)<1$, we can apply the test provided in Algorithm 2.2 to the matrix $I-B$, provided that $I-B$ is a $Z$-matrix.

\section{GROWTH FACTOR}

Taking into account Algorithm 2.2, we can define the following growth factor $g$ associated to the test presented there. If the test stops after calculating $b^{(k)}$ $(1 \leq k \leq n)$, then

$$
g:=\frac{\max \left\{\left|m_{i j}^{(r)}\right|,\left|b_{i}^{(r)}\right|,\left|b_{i}^{(k)}\right| ; 1 \leq i, j \leq n, 1 \leq r<k\right\}}{\max \left\{\left|m_{i j}\right| ; 1 \leq i, j \leq n\right\}} .
$$


If the test stops after calculating $M^{(k)}(1 \leq k \leq n-1)$, then

$$
g:=\frac{\max \left\{\left|m_{i j}^{(r)}\right|,\left|b_{i}^{(r)}\right| ; 1 \leq i, j \leq n, 1 \leq r \leq k\right\}}{\max \left\{\left|m_{i j}\right| ; 1 \leq i, j \leq n\right\}} .
$$

Obviously, $g \geq 1$.

Theorem 3.1. The growth factor $g$ associated to the test provided in Algorithm 2.2 for checking if a $Z$-matrix $M=\left(m_{i j}\right)_{1 \leq i, j \leq n}$ is a nonsingular $M$-matrix is $g \leq n-1$ and the equality can be achieved.

Proof. Using the notations of Algorithm 2.2, observe that, by the sign structure of the matrix $M=M^{(1)}$, the elements of $M e_{n}=b=b^{(1)}$ satisfy

$$
\frac{\max _{1 \leq i \leq n}\left\{\left|b_{i}^{(1)}\right|\right\}}{\max _{1 \leq i, j \leq n}\left\{\left|m_{i j}^{(1)}\right|\right\}} \leq n-1,
$$

and the equality can be achieved if $M$ has a row with a zero diagonal entry and with the remaining elements negative and all of them with maximal absolute value among all entries of $M$.

The chosen pivots $\tilde{m}_{r r}^{(r)}=m_{i_{r} r}^{(r)}$ are always positive. Hence, since the off-diagonal elements of $M$ are nonpositive, we add to each row a nonnegative multiple of the first one and the same property holds for the off-diagonal elements of $M^{(2)}$. Iterating the argument, it also holds that the obtained matrices $M^{(r)}$ are $Z$-matrices. The fact that in our elimination procedure we are always adding nonnegative multiples of the pivot row and the off-diagonal entries are nonpositive also implies that the positive diagonal elements are diminishing their value, so that they will be bounded above by the corresponding diagonal entries of $M$, and so, for each $p \geq 1$,

$$
\max _{1 \leq i \leq n}\left\{m_{i i}^{(p)}\right\} \leq \max _{1 \leq i \leq n}\left\{m_{i i}\right\} .
$$

Observe that, by our criterium for choosing the pivots, the corresponding component in the right-hand-side vector is always positive. Thus, the use of nonnegative multiples then implies that the negative components of the right-hand side are decreasing their absolute value, and so we have

$$
b_{i}^{(p)} \leq 0 \Rightarrow\left|b_{i}^{(p)}\right| \leq \max _{1 \leq i \leq n}\left\{\left|b_{i}^{(1)}\right|\right\} .
$$

Since the right-hand-side components are always the sum of the elements of the corresponding row of a $Z$-matrix, their positive components are bounded above by the corresponding diagonal element and so, by (3.2), we have

$$
b_{i}^{(p)}>0 \Rightarrow b_{i}^{(p)} \leq \max _{1 \leq i \leq n}\left\{m_{i i}\right\} .
$$

As in Algorithm 2.2, let $k$ be the positive integer corresponding to the step of Gaussian elimination such that the test ends. We finish the test after calculating either $b^{(k)}(k \leq n)$ or $M^{(k)}(k \leq n-1)$. From (3.1), (3.3) and (3.4), we derive for all $k \leq n$

$$
\frac{\max \left\{\left|b_{i}^{(k)}\right| ; 1 \leq i \leq n\right\}}{\max \left\{\left|m_{i j}\right| ; 1 \leq i, j \leq n\right\}} \leq n-1 .
$$

It remains to control the growth factor corresponding to the matrices $M^{(k)}$ $(2 \leq k \leq n-1)$. Let $P$ be the permutation matrix such that the first $k-1$ 
rows of the matrix $B:=P^{T} M P$ are the first $k-1$ pivot rows of $M$ chosen according to the pivoting strategy of the statement of Theorem 2.1. Then $B^{(k)}=M^{(k)}$ and, for each $r<k$, the first $r+1$ rows of $B^{(r+1)}$ coincide with the first $r+1$ rows of $M^{(r+1)}$. Again by the choice of the pivots, the first $r$ rows of $B^{(r+1)}=\left(b_{i j}^{(r+1)}\right)$ are strictly diagonally dominant. This and (3.2) imply for $l \leq r$ and any $j$ that

$$
\left|b_{l j}^{(r+1)}\right| \leq b_{l l}^{(r+1)}=m_{l l}^{(r+1)} \leq \max _{1 \leq i \leq n}\left\{m_{i i}\right\} .
$$

The mentioned strict diagonal dominance also implies that

$$
\sum_{j=r+1}^{n} \frac{\left|b_{r j}^{(r)}\right|}{\left|b_{r r}^{(r)}\right|}<1 .
$$

On the other hand, if $l>r$, let us consider $\left|b_{l j}^{(r+1)}\right|$. If $j \leq r$, then one has that

$$
b_{l j}^{(r)}=0 \text {. }
$$

Finally, let us consider the case $j>r$. Taking into account that we can write

$$
b_{l j}^{(r+1)}=b_{l j}^{(r)}-\frac{b_{r j}^{(r)}}{b_{r r}^{(r)}} b_{l r}^{(r)}
$$

and (3.6), one can deduce that

$$
\left|b_{l j}^{(r+1)}\right|<\left|b_{l, r}^{(r)}\right|+\left|b_{l j}^{(r)}\right| .
$$

Iterating the previous arguments, we obtain

$\left|b_{l j}^{(r+1)}\right|<\left|b_{l, r}^{(r)}\right|+\left|b_{l j}^{(r)}\right|<\left|b_{l, r-1}^{(r-1)}\right|+\left|b_{l r}^{(r-1)}\right|+\left|b_{l j}^{(r-1)}\right|<\cdots<\left|b_{l 1}\right|+\cdots+\left|b_{l, r}\right|+\left|b_{l j}\right|$ and so $\left|b_{l j}^{(r+1)}\right|<(r+1) \max _{i, j}\left|b_{i j}\right| \leq k \max _{i, j}\left|b_{i j}\right|=k \max _{i, j}\left|m_{i j}\right|$. Hence and using (3.5) and (3.7), we deduce that the growth factor of the elements of $M^{(2)}, \ldots, M^{(r+1)}$ is less than $k \leq n-1$ and the result follows.

The following example shows that if we check if a matrix is a nonsingular $M$ matrix by means of the signs of the pivots of GE without the pivoting strategy of our test, then the corresponding growth factor can be arbitrarily large.

Example 3.2. Let us consider the matrix

$$
M=\left(\begin{array}{ccc}
\varepsilon & 0 & -1 \\
-1 & 1 & 0 \\
0 & 0 & 1
\end{array}\right)
$$

$(0<\varepsilon<1)$ and assume that we want to know if it is a nonsingular $M$-matrix. If we apply GE without row exchanges in order to check the positivity of the pivots, we obtain the upper triangular matrix

$$
U=\left(\begin{array}{ccc}
\varepsilon & 0 & -1 \\
0 & 1 & -1 / \varepsilon \\
0 & 0 & 1
\end{array}\right)
$$

and so the corresponding growth factor is $1 / \varepsilon$. However, if we apply the test provided in Algorithm 2.2, we obtain

$$
b^{(1)}=\left(\begin{array}{c}
\varepsilon-1 \\
0 \\
1
\end{array}\right), \quad \tilde{M}^{(1)}=\left(\begin{array}{ccc}
1 & 0 & 0 \\
0 & 1 & -1 \\
-1 & 0 & \varepsilon
\end{array}\right), \quad \tilde{b}^{(1)}=\left(\begin{array}{c}
1 \\
0 \\
\varepsilon-1
\end{array}\right)
$$


and, after the first step of GE,

$$
b^{(2)}=\left(\begin{array}{l}
1 \\
0 \\
\varepsilon
\end{array}\right), \quad M^{(2)}=\left(\begin{array}{ccc}
1 & 0 & 0 \\
0 & 1 & -1 \\
0 & 0 & \varepsilon
\end{array}\right)
$$

and the growth factor is $g=1$ and it does not depend on $\varepsilon$.

\section{REFERENCES}

[1] A. A. Ahac and D. D. Olesky, A stable method for the $L U$ factorization of $M$-matrices, SIAM J. Alg. Disc. Math. 7 (1986), 368-378. MR 87i:65035

[2] A. Berman and R. J. Plemmons, Nonnegative matrices in the mathematical sciences, SIAM, Philadelphia, 1994. MR 95e:15013

[3] K. Fan, Note on M-matrices, Quart. J. Math. Oxford Ser. (2) 11 (1961), 43-49. MR 22:8024

[4] G. H. Golub and C. F. Van Loan, Matrix computations (3rd edition), The John Hopkins University Press, 1996. MR 97g:65006

[5] G. H. Golub and J. M. Ortega, Scientific computing and differential equations. An introduction to numerical methods, Academic Press, Boston, 1992. MR 92f:65002

[6] A. N. Malyshev, A note on the stability of Gauss-Jordan elimination for diagonally dominant matrices, Computing 65 (2000), 281-284. MR 2001k:65055

[7] J. M. Ortega, Numerical Analysis. A second course, SIAM, Philadelphia, 1990. MR 90k:65005

[8] J. M. Peña, Pivoting strategies leading to diagonal dominance by rows, Numer. Math. 81 (1998), 293-304. MR 2000j:65037

Departamento de Matemática Aplicada, Universidad de Zaragoza, 50006 Zaragoza, SPAIN

E-mail address: jmpena@posta.unizar.es 